Journal for ImmunoTherapy of Cancer

\title{
Dual but not single PD-1 or TIM-3 blockade enhances oncolytic virotherapy in refractory lung cancer
}

\author{
Fan Sun, ${ }^{1,2}$ Zong Sheng Guo, ${ }^{1,3}$ Alyssa D Gregory, ${ }^{4}$ Steven D Shapiro, ${ }^{4}$ \\ Gutian Xiao, ${ }^{1,2}$ Zhaoxia Qu (D) ${ }^{1,2}$
}

To cite: Sun F, Guo ZS, Gregory AD, et al. Dual but not single PD-1 or TIM-3 blockade enhances oncolytic virotherapy in refractory lung cancer. Journal for ImmunoTherapy of Cancer 2020;8:e000294. doi:10.1136/jitc-2019-000294

- Additional material is published online only. To view please visit the journal online (http://dx.doi.org/10.1136/jitc2019-000294).

Accepted 07 April 2020

Check for updates

(C) Author(s) (or their employer(s)) 2020. Re-use permitted under CC BY-NC. No commercial re-use. See rights and permissions. Published by BMJ.

${ }^{1}$ UPMC Hillman Cancer Center, University of Pittsburgh Medical Center, Pittsburgh, Pennsylvania, USA

${ }^{2}$ Department of Microbiology and Molecular Genetics, University of Pittsburgh School of Medicine, Pittsburgh, Pennsylvania, USA

${ }^{3}$ Department of Surgery, University of Pittsburgh School of Medicine, Pittsburgh,

Pennsylvania, USA

${ }^{4}$ Department of Medicine, University of Pittsburgh Medical Center, University of Pittsburgh, Pittsburgh, Pennsylvania, USA

\section{Correspondence to}

Dr Zhaoxia Qu; quz@upmc.edu

Dr Gutian Xiao;

xiaog2@upmc.edu

\section{ABSTRACT}

Background Programmed cell death 1 (PD-1)/ programmed death ligand 1 (PD-L1) blockade therapy fails in the majority of patients with cancer. Oncolytic viruses represent a new class of therapeutic agents, yet the therapeutic efficacy is still disappointing. Moreover, intratumoral injection of viruses is the main approach and preclinical studies mainly employ syngeneic or xenograft models.

Methods Use an endogenous mouse lung cancer model that faithfully recapitulates human lung cancer, and various in vivo, ex vivo and in vitro assays, to investigate the efficacy, mechanism of action and resistance of systemically administered oncolytic vaccinia virus (oVV), immunotherapy and their combination, to find an effective therapy for refractory lung cancer.

Results Resembling human lung cancers, the majority of which are largely resistant to PD-1/PD-L1 blockade and with decreased PD-L1 expression and T-cell activation by our analysis, urethane-induced endogenous lung tumors in mice show reduced PD-L1 expression, low tumor-infiltrating lymphocytes and innate resistance to PD-1/PD-L1 blockade. Intravenous administration of oVV has efficacy and synergizes with simultaneous but not single blockade of PD-1 and T-cell immunoglobulin and mucin-domain containing-3 (TIM-3) in this cancer model. Besides direct tumor cell killing, oV induces T-cell lung recruitment, tumor infiltration, along with expression of PD-1 and TIM-3 on T cells and PD-1 and TIM-3 ligands on tumor cells and tumor-associated immune cells. Blockade of PD-1 or TIM-3 also causes their mutual induction on T cells.

Conclusions While systemic administration of oW shows efficacy in lung cancer by killing tumor cells directly and recruiting and activating $T$ cells for indirect tumor killing, its induction of PD-1 and TIM-3 on T cells and PD- 1 and TIM-3 ligands on tumors and tumor-associated immune cells as well as mutual induction of PD-1 or TIM-3 on T cells by their blockade restricts the efficacy of oV or its combination with single PD-1 or TIM-3 blockade. The triple combination therapy is more effective for refractory lung cancer, and possibly other cold cancers as well.

\section{BACKGROUND}

Blockade of the ligation of the immune checkpoint PD-1 (also known as CD279) with PD-L1 (also known as B7-H1 or CD274) using PD-1 or PD-L1 antibody has recently been approved by the Food and Drug Administration as a standard treatment for several cancers, including lung cancer, the leading cause of cancer deaths in both men and women. $^{1-3}$ In general, this revolutionary immunotherapy works better against 'hot' tumors, which have abundant infiltrated $\mathrm{T}$ cells, strong immunogenicity and sufficient PD-L1 expression. Unfortunately, most tumors are 'cold' and do not respond. ${ }^{4-7}$ Even among the most responsive cancer types such as lung cancer, the response rate is only about $20 \%$ and resistance may occur after an initial response. ${ }^{8-10}$ Hence, there is a need to explore combination therapies to expand the benefit of this innovative immunotherapy to a greater number of patients.

Oncolytic viruses (OVs) may be an ideal candidate to combine with PD-1/PD-L1 blockade to improve cancer treatment. ${ }^{11-15}$ Currently, several dozen OVs, most of which are armed with granulocyte-macrophage colony-stimulating factor (GM-CSF) or other cytokines, are being evaluated for cancer treatment. Although their safety profile is encouraging, the antitumor efficacy of OVs alone appears to be disappointing. ${ }^{12} 131617$ In addition, local administration and in particular intratumoral injection of OVs, which is the main approach used in both clinical and preclinical studies, also limits their application, particularly for tumors difficult to access, such as lung cancer.

Of note, viruses, including OVs, induce PD-L1 within sites of infection including the tumor microenvironment (TME), and PD-1 on $\mathrm{T}$ cells to terminate T-cell activity for immune escape. ${ }^{18-20}$ While this PD-L1/ PD-1-mediated immune tolerance commonly used by viruses and tumors may account for the disappointing antitumor activity of OVs, it makes OVs an ideal tool to turn cold tumors hot and sensitive to PD-1/PD-L1 blockade, 
particularly given additional roles of OVs in inducing T-cell tumor infiltration and tumor immunogenicity. PD-1 blockade should mitigate the PD-L1/PD-1-mediated tumor-promoting function of OVs, thus OVs and PD-1/ PD-L1 therapies should synergize to promote tumor clearance. In this regard, virus-associated tumors are known to respond at high rates to PD-1 pathway blockade. ${ }^{21}$

Here, we test whether intravenous administration of vaccinia virus with Double Deletion (vvDD) alone, a genetically modified oncolytic vaccinia virus with tumorselectivity rendered by double deletion of the thymidine kinase gene and the vaccinia growth factor gene, ${ }^{16} 1722$ has efficacy in refractory lung cancer in an endogenous mouse tumor model that faithfully recapitulates human lung cancer. We also examine whether and how vvDD synergizes with blockade of PD-1 and/or TIM-3 (also known as HAVCR2 or CD366), another immune checkpoint that has not yet been approved for clinical use. ${ }^{2324}$

\section{METHODS}

\section{Animals and cell lines}

Mice of BALB/c, FVB/N and C57BL/ 6 strains were originally from The Jackson Laboratory, and maintained under pathogen-free conditions and used according to protocols approved by Institutional Animal Care and Use Committee of the University of Pittsburgh. Mouse lung cancer cell lines Madison 109 (MAD109), Lewis lung carcinoma (LLC) and LAP0297 were kindly provided by Alan L Epstein (University of Southern California), Per H. Basse/Stephen H. Thorne (University of Pittsburgh) and Dai Fukumura/Peigen Huang (Massachusetts General Hospital and Harvard Medical School), respectively. ${ }^{25}{ }^{26}$ Human cancer cell lines were described before ${ }^{27}$ and were cultured at $37^{\circ} \mathrm{C}$ in humidified $5 \%$ $\mathrm{CO}_{2}$ incubator. Culture medium were supplemented with $10 \%$ fetal bovine serum (FBS) and penicillin/streptomycin (Invitrogen, Carlsbad, CA, USA). H23, H460, H727, H1975, H2122 cells were cultured in Roswell Park Memorial Institute (RPMI) 1640 medium . Calu-1 cells were cultured in McCoy's 5a. Calu-6 and SK-LU-1 cells were cultured in Eagle's minimum essential medium . All the other cell lines were cultured in Dulbecco's modified Eagle's medium (DMEM). Cell lines were authenticated by short tandem repeat profiling, and tested for Mycoplasma (last tested in October 2018, IDEXX BioAnalytics).

\section{Viruses and viral infection in vitro}

Recombinant Vaccinia virus (Western Reserve strain) vvDD was described previously. ${ }^{22}$ Mouse or human cells $\left(4 \times 10^{5}\right)$ were seeded in six-well plates overnight and then infected with vvDD at MOI of 1.0 in $0.6 \mathrm{~mL}$ of $2 \%$ FBScontaining DMEM for 2 hours. Growth medium ( $1.4 \mathrm{~mL})$ was added and the cells were collected around 72 hours post virus infection.
In vitro tumor antigen-dependent T-cell activation and tumor cell killing

As previously described ${ }^{28}$ bone marrow-derived dendritic cells (BMDCs) from BALB/c mice were pulsed with the lysates (filtered with Amicon Ultra-15 50K Centrifugal Filter, MilliporeSigma, Burlington, Massachusetts, USA) of the detached MAD109 cells 18 hours post in vitro infection with vvDD (1:3 ratio). As controls, PBS mock-treated MAD109 cells as well as wDDD-infected or PBS mocktreated normal lung epithelial cells from BALB/c mice were also included in parallel. Pulsed BMDCs were then cocultured with splenic $\mathrm{CD}^{+} \mathrm{T}$ cells from BALB/c mice (1:5 ratio) in the presence of interleukin-2 $(50 \mathrm{U} / \mathrm{mL})$ for 4 days, followed by fluorescent activated cell sorting (FACS) analysis to detect interferon $\gamma(\mathrm{IFN} \gamma)^{+}$, granzyme $\mathrm{B}^{+}$and $\mathrm{CD} 69^{+} \mathrm{CD}^{+} \mathrm{T}$ cells. T cells isolated from BMDC coculture were further cocultured with MAD109 or LLC cells stably expressing luciferase (20:1 ratio) for 4 hours, followed by luciferase activity measure in the supernatant (indication of cell apoptosis, as luciferase can be released into the medium only after cell death).

\section{Lung tumor models}

Urethane model: As described before ${ }^{29}$ female FVB/N mice aged $6-8$ weeks were intraperitoneally (i.p.) injected with urethane $(1 \mathrm{~g} / \mathrm{kg}$ body weight, Sigma-Aldrich, St. Louis, Missouri, USA) once a week for six consecutive weeks. Mice were then treated with vvDD (intravenous injection, $10^{8} \mathrm{pfu} / \mathrm{mouse}$ ), PD-1 and/or TIM-3 neutralizing antibodies (i.p. injection, 3 times/week, $200 \mu \mathrm{g} /$ mouse/time for PD-1 antibody, $100 \mu \mathrm{g} / \mathrm{mouse} /$ time for TIM-3 antibody, BioXCell, West Lebanon, New Hampshire, USA). All mice were sacrificed for lung tumor examinations 2 weeks post vvDD treatment. Surface tumors in mouse lungs were counted by three blinded readers under a dissecting microscope, and tumor diameters were measured by microcalipers. The volume of each tumor was calculated as $(\pi / 6) \times \mathrm{D}^{3}$ (D, tumor diameter). Tumor burden of each mouse was calculated as the sum of the volumes of each tumor in all the lung lobes of that mouse.

Syngeneic model: essentially as described before, ${ }^{30}$ 6-week-old female FVB/N or BALB/c mice were challenged subcutaneously (s.c.) in the right flank with $10^{6}$ LAP0297 or MAD109 cells, respectively. Eight days posttumor cell inoculation, tumors were injected with vvDD $\left(10^{8} \mathrm{pfu} /\right.$ mouse $)$ or PBS. Tumors were measured every 2 days post-tumor cell injection. The volume of each tumor was calculated as $(\pi / 6) \mathrm{x}$ width $^{2} \mathrm{x}$ length. All the mice were sacrificed 16 days post-tumor cell injection and tumor masses were dissected for further analysis. The effects of PD-1 and TIM-3 blockade alone or together with vvDD treatment on syngeneic LAP0297 tumors and mouse survival were also examined. The LAP0297 tumors were injected with vvDD $\left(10^{8} \mathrm{pfu} /\right.$ mouse $)$ or PBS 8 days post-tumor cell inoculation, and/or treated with PD-1 and TIM-3 neutralizing antibodies or control PBS starting 9 days post-tumor cell inoculation (i.p. injection 
every other day, $200 \mu \mathrm{g} /$ mouse/time for PD-1 antibody, $100 \mu \mathrm{g} / \mathrm{mouse} /$ time for TIM-3 antibody, BioXCell, West Lebanon, New Hampshire, USA).

\section{Flow cytometry (FACS) analysis}

The cells were incubated with the antibodies against cell surface antigens after blocked with $\alpha \mathrm{CD} 16 / \mathrm{CD} 32$. The cells were then fixed with paraformaldehyde $(2 \%)$, permeablized and incubated with antibodies against intracellular antigens when needed. For IFN $\gamma$ staining, cells were treated with phorbol 12-myristate 13-acetate (50 $\mathrm{ng} / \mathrm{mL})$, ionomycin $(1 \mu \mathrm{M})$, brefeldin $\mathrm{A}(3 \mu \mathrm{g} / \mathrm{mL})$ and monensin $(2 \mu \mathrm{M})$ for 4 hours before they were stained for FACS analysis. Data were acquired and analyzed by Accuri C6 (BD Biosciences, Bedford, Massachusetts, USA) as described previously ${ }^{31}$ or acquired by LSRFortessa (BD Biosciences) and analyzed by FlowJo software. Immune cells $\left(\mathrm{CD} 45^{+}\right)$were gated with the indicated markers as follows. Lymphocytes were gated for $\mathrm{CD}^{+} \mathrm{T}$ cell $\left(\mathrm{CD}^{+} \mathrm{CD}^{+} \mathrm{CD}^{-}\right), \mathrm{CD}^{+} \mathrm{T}^{+}$cell $\left(\mathrm{CD}^{+} \mathrm{CD} 4{ }^{-} \mathrm{CD} 8^{+}\right)$, natural killer (NK) cell $\left(\mathrm{NKp} 46^{+} \mathrm{CD} 3^{-}\right)$and $\mathrm{B}$ cell $\left(\mathrm{B}^{2} 20^{+} \mathrm{CD}^{-}\right.$ ). Myeloid cells were gated sequentially as follows:

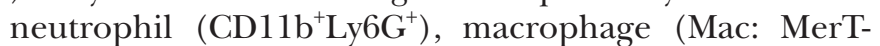

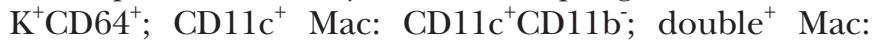

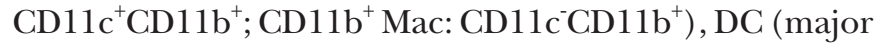
histocompatibility complex class II (MHC-II $)^{+} \mathrm{CD} 11 \mathrm{c}^{+}$), monocyte (Mono: CD11 b ${ }^{+} S^{\text {lo }}$; inflammatory monocyte (iMono): Ly6 $\mathrm{C}^{\text {hi }}$; resident monocyte (rMono): Ly6 $\mathrm{C}^{\mathrm{lo}}$ ).

\section{Histology and immunohistochemistry assays}

As described previously, ${ }^{32} 3$ mouse or human tissues were excised, fixed in formalin, embedded in paraffin, and cut into 5- $\mu \mathrm{m}$-thick sections. Sections were stained with $\mathrm{H} \& \mathrm{E}$, or subjected to sequential incubations with different primary antibodies, biotinylated secondary antibody, and streptavidin-HRP. Antibodies used for histology and FACS assays were listed in online supplementary additional file 2: online supplementary table S1.

\section{Quantitative PCR analysis}

The indicated tissues or cells were subjected to RNA extraction, RNA reverse transcription and real-time PCR as described. ${ }^{34}{ }^{35}$ Primer pairs used for quantitative PCR were listed in online supplementary additional file 2: online supplementary table S2.

\section{STATISTICAL ANALYSIS}

Student's t-test (two tailed, unpaired unless otherwise specified) was used to assess significance of differences between two groups. Log-rank test was used to compare survival between treatment groups. All bars in figures represent means \pm SEM. The sample values for FACS and immunohistochemistry (IHC) quantifications are the numbers of mice and tumors used for the assays, respectively. The $\mathrm{p}$ values are indicated as $* \mathrm{p}<0.05, * * \mathrm{p}<0.01$, $* * * p<0.001, * * * * p<0.0001, n s$, not statistically significant.
The $\mathrm{p}$ values $<0.05$ and $<0.01$ are considered statistically significant and highly statistically significant, respectively.

\section{RESULTS \\ Downregulation of PD-L1 in the majority of human lung cancers}

To determine why most human lung cancers do not respond well to PD-1 pathway blockade therapy, we analyzed The Cancer Genome Atlas (TCGA) databases for PD-L1 expression in human lung cancers. PD-L1 expression level in tumor tissues is one most commonly used and reliable biomarker for PD-1 and PD-L1 blockade therapy. ${ }^{6-9}$ Whereas PD-L1 was upregulated in about $11 \%$ of human lung cancers, it was downregulated in more than $60 \%$ of all human lung cancer cases compared with normal lung tissues (figure 1A; online supplementary additional file 1: online supplementary figure S1a-c). This finding was confirmed with IHC staining of two independent tumor tissue arrays demonstrating that PD-L1 was only detected in $\sim 25 \%-30 \%$ of human lung tumors. ${ }^{36}$ Analysis of European Molecular Biology Laboratory-European Bioinformatics Institute (EMBL-EBI) Expression Atlas databases also revealed that PD-L1 was repressed in about $60 \%$ of human lung cancer cell lines (figure 1B). These findings were further validated in our human lung cancer and cell line studies (figure 1C-E; online supplementary additional file 1: online supplementary figure S1d,e).

To figure out the mechanism of PD-L1 repression in lung cancer, we analyzed the TCGA data for the methylation status of the $p d-l 1$ promoter in human lung cancers. In line with our recent studies showing that all three functional DNA methyltransferases are increased in human lung cancers, ${ }^{37}$ we found that the methylation of the $p d-l 1$ promoter was increased in human lung cancers compared with normal lung tissues (online supplementary additional file 1: online supplementary figure S1f). Consistently, the demethylating agent 5 -aza-dC induced expression of PD-L1 in lung cancer cells in vitro (online supplementary additional file 1: online supplementary figure S1g). We also found that T-cell activation and IFN signature gene expression was downregulated in human lung cancers and that IFN $\gamma$ induced PD-L1 expression in lung cancer cells ${ }^{37} 38$ (online supplementary additional file 1: online supplementary figure S1h-j). These data indicate that PD-L1 downregulation in lung cancer involves its promoter epigenetic repression and inflammation downregulation within the TME.

Similar to PD-L1, PD-L2 (also known as B7-DC or CD273), the other known ligand of PD-1, was also suppressed in most lung cancers (online supplementary additional file 1: online supplementary figure S2). These data together suggest that resistance to PD-1 blockade in most lung cancer patients may involve the downregulation of PD-L1 and PD-L2. 

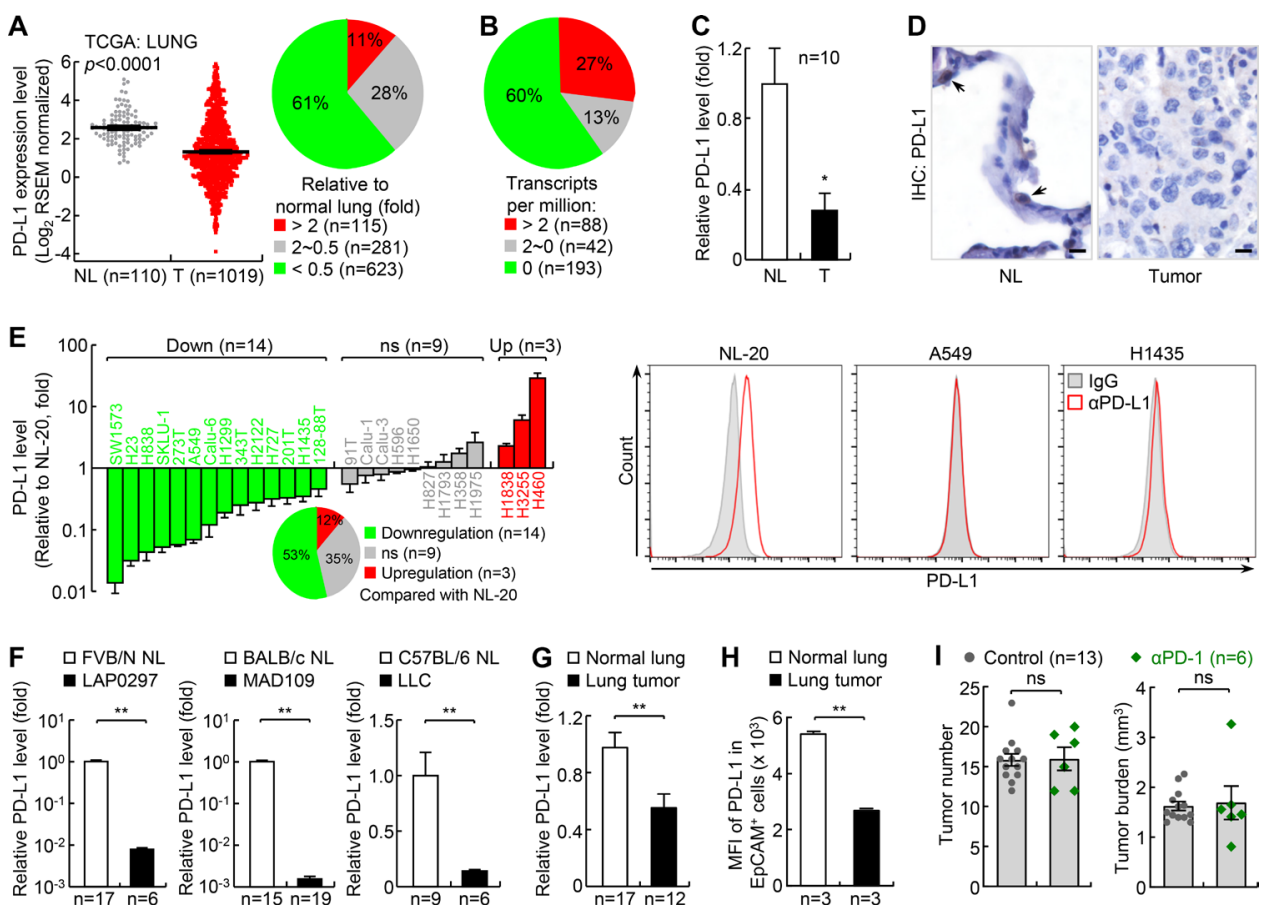

Figure 1 PD-L1 is downregulated in the majority of human lung cancers and mouse lung cancers, and mouse lung cancers induced by urethane are largely resistant to PD-1 blockade therapy. (A) TCGA data showing PD-L1 downregulation in human lung cancer. Left: gray dots and red dots stand for normal lung (NL) tissues and lung tumor (T) tissues, respectively. Right: PDL1 expression profile in lung cancer tissues. Sample numbers are indicated. (B) EMBL-EBI data showing PD-L1 downregulation in human lung cancer cell lines. (C) Quantitative RT-PCR (qPCR) analysis showing PD-L1 downregulation in human lung cancer. Matched normal lung tissues from the same patients were used as controls. (D) IHC assay showing PD-L1 downregulation in human lung cancer. Scale bar, $20 \mu \mathrm{m}$. (E) qPCR and FACS analysis showing PD-L1 downregulation in most human lung cancer cell lines $(n=3)$. NL-20 is a normal human lung epithelial cell line; others are human lung cancer cell lines. (F) qPCR analysis showing PD-L1 downregulation in mouse lung cancer cell lines. $(G, H)$ qPCR $(G)$ and FACS $(H)$ analysis showing PD-L1 downregulation in mouse primary lung cancers induced by urethane. (I) Urethane model showing large resistance of lung cancer to PD-1 blockade therapy. FACS, fluorescent activated cell sorting; MFI, mean fluorescence intensity; ${ }^{*} \mathrm{p}<0.05$; ${ }^{* *} \mathrm{p}<0.01$; ns, not significant; PD-1, programmed cell death 1; PD-L1, programmed death ligand 1; RSEM, RNA-Seq by Expectation Maximization; TCGA, The Cancer Genome Atlas.

\section{Establishment of a reliable lung cancer model for studying and improving PD-1 therapy}

Similar to our human studies, we found that PD-L1 was downregulated in mouse lung cancer cell lines MAD109, LLC and LAP0297, which were originally derived from spontaneous lung tumors developed in BALB/c, C57BL/6 and FVB/N mice, respectively (figure 1F). PD-L1 was also downregulated in mouse primary lung cancers induced by ethyl carbamate (also called urethane), a chemical carcinogen present in fermented food, alcoholic beverage and cigarette smoke (figure $1 \mathrm{G}, \mathrm{H}$ ). It is noteworthy that murine lung cancer induced by urethane faithfully recapitulates human lung cancer, and in particular adenocarcinoma, the most common type of lung cancer that accounts for about $40 \%$ of all lung cancers. ${ }^{27-29} 373940$ Moreover, our recent studies have shown that PD-L1 expression can be induced in mouse lung tumor cells both in vitro and in vivo by epigenetic drugs or through immune activation by chemotherapeutic drugs. ${ }^{37}$ These data demonstrate that mouse lung cancers, like their human counterparts, also share PD-L1 downregulation.

Based on these findings, we tested whether mouse lung cancers induced by urethane, like human lung cancers with low PD-L1 expression, are also resistant to PD-1 blockade. As expected, they were largely resistant to PD-1 blockade, with no significant changes in both tumor number and tumor burden after PD-1 antibody treatment (figure 1I; online supplementary additional file 1: online supplementary figure S3). Urethane-induced lung cancer in mice thus provides a clinically reliable model of refractory lung cancer that can be used to test new human lung cancer therapies, particularly those that can overcome PD-1 blockade resistance and synergize with PD-1 blockade therapy.

\section{Efficacy of vvDD alone in the treatment of refractory lung cancer}

Mice with lung tumors induced by urethane were intravenous injected with vvDD or PBS. Two weeks post vvDD injection, mice were sacrificed, and lung tissues were collected. Compared with the PBS mock-treated group, vvDD-treated mice had a significantly reduced tumor burden in their lungs, although tumor numbers were similar in those mice (figure 2A). Thus, systemic administration of vvDD alone shrinks but does not ablate individual lung nodules in this model of refractory lung cancer. It is 

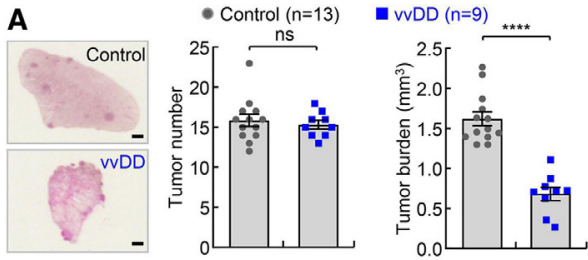

C
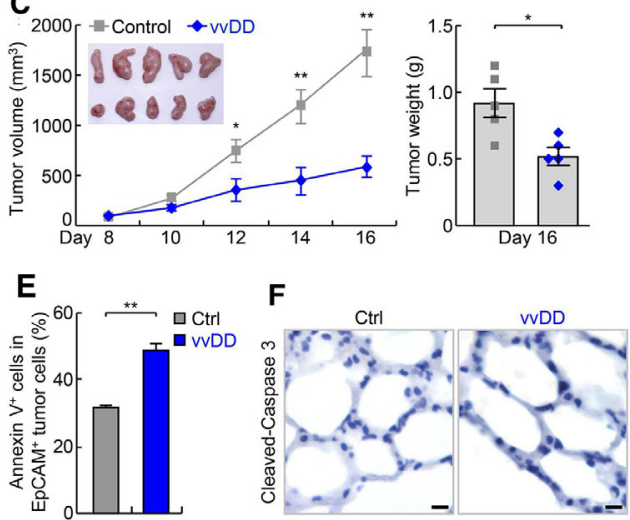
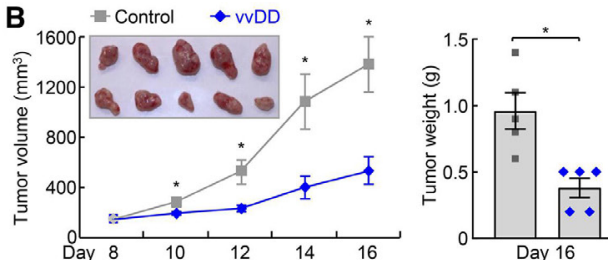

D
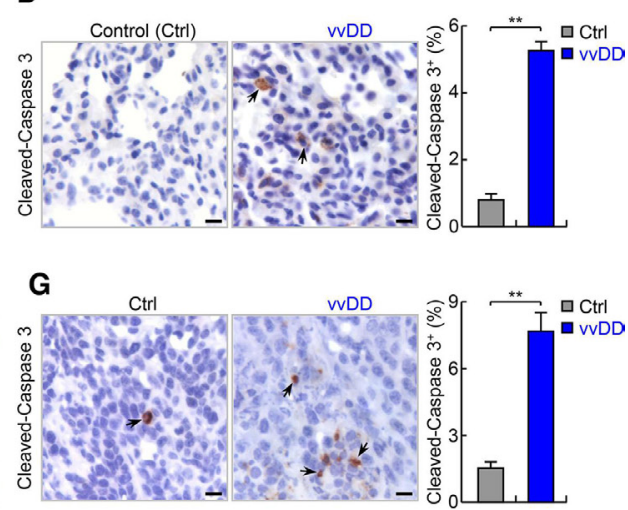

Figure 2 vvDD shows efficacy in refractory lung cancer in endogenous and syngeneic mouse lung tumor models. (A) Urethane model showing efficacy of vvDD intravenous administration in refractory lung cancer. scale bar, $1 \mathrm{~mm}$. (B) Syngeneic MAD109 lung cancer model showing efficacy of vvDD intratumoral injection in refractory lung cancer $(n=5)$. In tumor image, top row: control; bottom row: vvDD. (C) Syngeneic LAP0297 lung cancer model showing efficacy of vvDD intratumoral injection in refractory lung cancer $(n=5)$. In tumor image, top row: control; bottom row: vVDD. (D-F) IHC and FACS showing increased apoptosis by vvDD treatment in tumor cells $((D), n=9$ and $(E), n=3)$, but no obvious apoptosis in normal lung cells $(F)$ in urethane model. Scale bar, $10 \mu \mathrm{m}$. (G) IHC showing increased tumor cell apoptosis by vVDD treatment in syngeneic MAD109 model $(n=8)$. Scale bar, $10 \mu \mathrm{m}$. FACS, fluorescent activated cell sorting; IHC, immunohistochemistry; ${ }^{*} p<0.05,{ }^{* *} p<0.01,{ }^{* * *} p<0.001$, ${ }^{* * *} \mathrm{p}<0.0001$; ns, not significant; vvDD, vaccinia virus with double deletion.

noteworthy that in line with previous human clinical trial studies showing the promising safety of systemic administration of voDD,${ }^{17}$ no significant side effect of vvDD treatment was observed in our mouse studies, as evidenced by no significant changes in mouse weight by the treatment (online supplementary additional file 1: online supplementary figure S4a).

We next repeated the experiment using vvDD versus PBS intratumoral injection in syngeneic MAD109 lung cancer model. Tumors form at fast rates in syngeneic models, and importantly, can be monitored easily and daily. Eight days after subcutaneous inoculation of MAD109 cells, vvDD or PBS was injected intratumorally. Compared with PBS treatment, vvDD treatment led to a significantly reduced tumor burden starting from 2 days post virus treatment (figure 2B). The difference became larger over time, with PBS-treated control tumors continuing to grow, while those treated with vvDD almost stopping growing during the remaining experimental period. Similar results were also obtained in syngeneic LAP0297 lung cancer model (figure 2C). These data further supported the efficacy of vvDD in treating refractory lung cancer.

Consistent with the anti-lung cancer activity of vvDD, significantly higher degrees of apoptosis were detected in lung tumors from wDD-treated mice compared with PBStreated control mice in both endogenous and syngeneic models (figure 2D-G). No obvious apoptosis was detected within normal lung tissues in either group (figure 2F). Of note, vDD was only detected in lung tumors but not in normal lung tissues of the vvDD-treated mice, supporting vvDD selective replication in tumor cells in vivo (online supplementary additional file 1: online supplementary figure S4b). In addition, higher apoptosis was detected in lung cancer cells positive with vvDD in comparison to those negative with vvDD, suggesting direct killing of tumor cells by vvDD infection (online supplementary additional file 1: online supplementary figure S4c). To validate the in vivo studies, we examined in vitro whether vvDD selectively kills mouse and human lung cancer cell lines with low PD-L1 expression. Infection with voD indeed resulted in much more death of both mouse and human lung cancer cells but had no effect on normal lung epithelial cells, further supporting a selective tumorkilling activity of vvDD (figure 3A).

In addition to direct oncolysis, another presumptive function of OVs is to induce tumor-specific immunity for tumor killing. Thus, we also performed the in vitro assays of lung tumor antigen-dependent T-cell activation and tumoricidal activity. ${ }^{28}$ Whereas DCs pulsed with vvDDinfected normal lung epithelial cells or uninfected lung cancer cells failed to activate T cells, DCs pulsed with vvDD-infected lung cancer cells induced strong tumorspecific activation and cytotoxicity of T cells (figure 3B,C). Consistent with these in vitro studies, significantly more $\mathrm{CD}^{+}$and $\mathrm{CD}^{+} \mathrm{T}$ cells expressing the T-cell activation markers IFN $\gamma$ and $\mathrm{CD} 44$ were detected in the lung and 

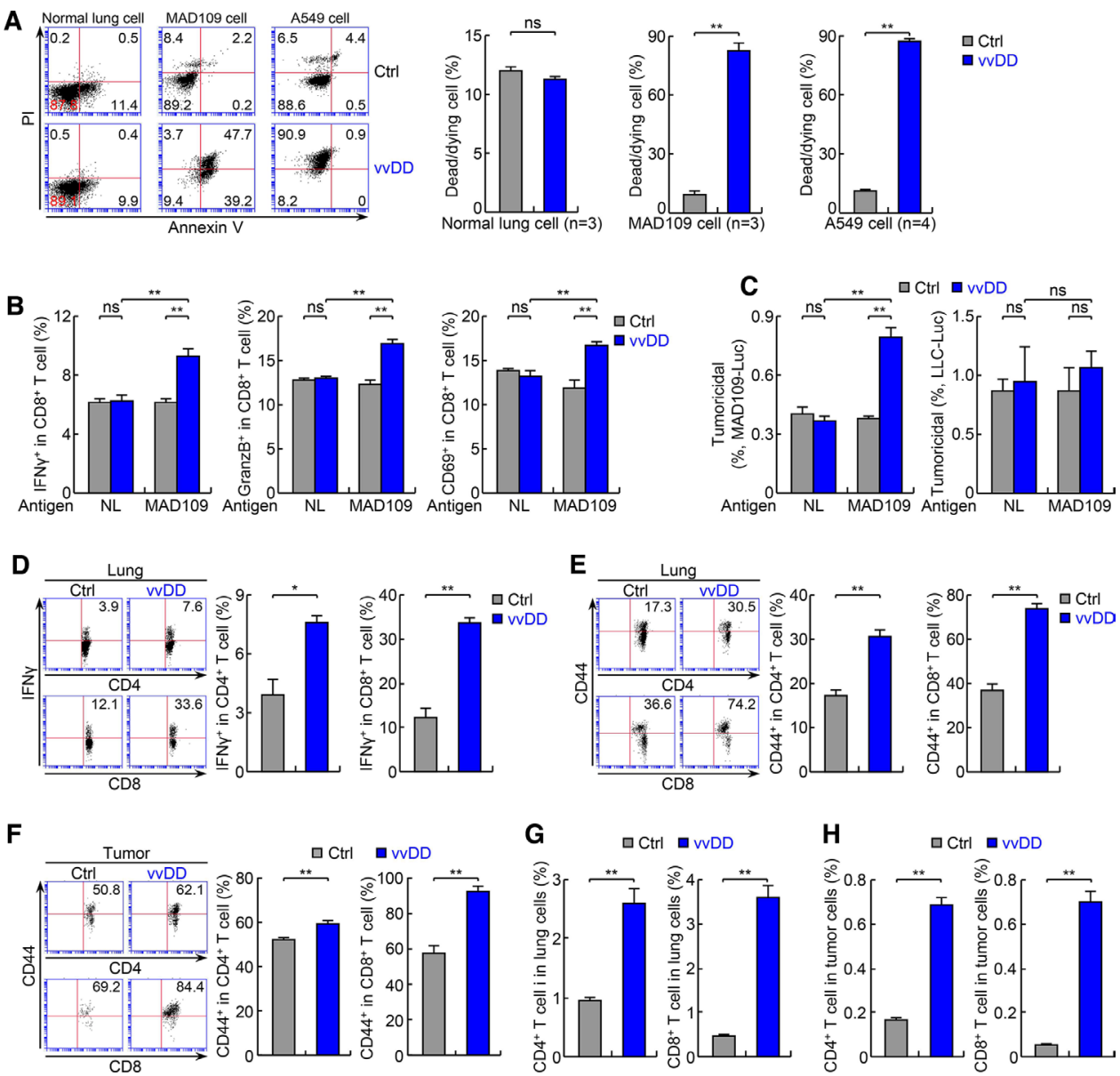

Figure 3 The antitumor actions of vvDD involve direct oncolysis and indirect immune killing of tumor cells. (A) In vitro infection and FACS assays showing selective killing of mouse and human lung tumor cells but not normal cells by vvDD. (B) In vitro lung tumor antigen-dependent T-cell activation assays showing tumor-specific T-cell activation by vvDD $(n=3)$. Normal lung cells: NL. (C) In vitro tumoricidal assay using T cells from (B) showing tumor antigen-dependent killing of T-cell specific MAD109 cells (left panel) but not T-cell non-specific LLC cells (right panel) ( $n=3$ ). (D, E) FACS assays of IFN $\gamma$ and CD44 showing increased lung $C D 4^{+}$and $C D 8^{+}$T-cell activation by vvDD treatment in urethane model $(n=3)$. $(F)$ FACS assay of $C D 44$ showing increased activation of tumor-infiltrating $C D 4^{+}$and $C D 8^{+} T$ cells by vvDD treatment in urethane model $(n=4)$. (G) FACS assays showing increased lung $\mathrm{CD}^{+}$and $\mathrm{CD} 8^{+} \mathrm{T}$ cells by vvDD treatment in urethane model $(\mathrm{n}=6)$. (H) FACS assays showing increased tumorinfiltrating $\mathrm{CD}^{+}$and $\mathrm{CD} 8^{+} \mathrm{T}$ cells by vvDD treatment in urethane model $(\mathrm{n}=6)$. FACS, fluorescent activated cell sorting; IFN $\gamma$, interferon- $\gamma$, LLC, Lewis lung carcinoma; ${ }^{*} \mathrm{p}<0.05$, ${ }^{* *} \mathrm{p}<0.01$; ns, not significant; vvDD, vaccinia virus with double deletion.

in particular lung tumors of mice treated with vvDD (figure 3D-F). In vivo vvDD treatment also increased both $\mathrm{CD} 4^{+}$and $\mathrm{CD} 8^{+} \mathrm{T}$-cell lung recruitment and tumor infiltration (figure $3 \mathrm{G}, \mathrm{H}$ ). It is worthy to note that in this endogenous model lung tumors contained significantly decreased tumor-infiltrating lymphocytes (TILs) compared with normal lung tissues (figure 3G,H; online supplementary additional file 1: online supplementary figure S4d), another major characteristic of cold tumors resistant to PD-1 blockade immunotherapy. Increased tumor infiltration of $\mathrm{CD}^{+} \mathrm{T}$ cells by vvDD treatment was also observed in the syngeneic lung cancer models (online supplementary additional file 1: online supplementary figure S4e). Besides $\mathrm{T}$ cells, vvDD treatment also increased tumor infiltration of many other immune cells, such as macrophages, DCs, monocytes, NK cells and B cells (online supplementary additional file 1: online supplementary figure S4f). Interestingly, vvDD treatment upregulated the expression of the antigen presenting MHC-II on tumor-infiltrating DCs and macrophages as well as the expression of the antibody Fc-gamma receptor (also known as CD64) on tumor infiltrating DCs (online supplementary additional file 1: online supplementary figure S4g). Altogether, these data suggest that vvDD exerts its antitumor activity through both direct oncolysis and indirect immune killing of tumor cells.

\section{No synergy between vvDD and PD-1 or TIM-3 antibody in the treatment of refractory lung cancer}

In addition to inducing a strong T-cell activation in the TME, in vivo vvDD treatment also led to increased PD-1 expression on both $\mathrm{CD} 4^{+}$and $\mathrm{CD} 8^{+} \mathrm{T}$ cells in the lung and in particular on $\mathrm{CD}^{+} \mathrm{T}$ cells in lung tumors (figure 4A,B). Of note, increased percentages of PD- $1^{+} \mathrm{T}$ cells, of both the $\mathrm{CD} 4^{+}$and $\mathrm{CD} 8^{+}$subsets, were observed in the TME compared with adjacent normal lung either 

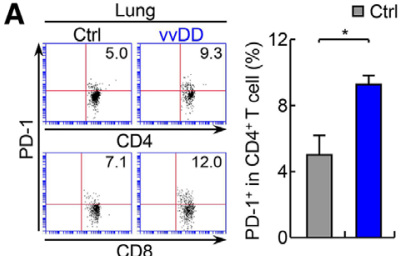

$\square$ Ctrl $\square \mathrm{wDD}$
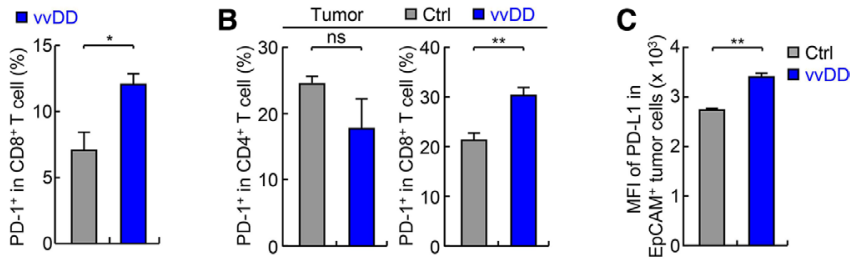

D

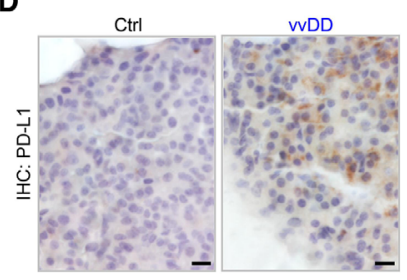

E
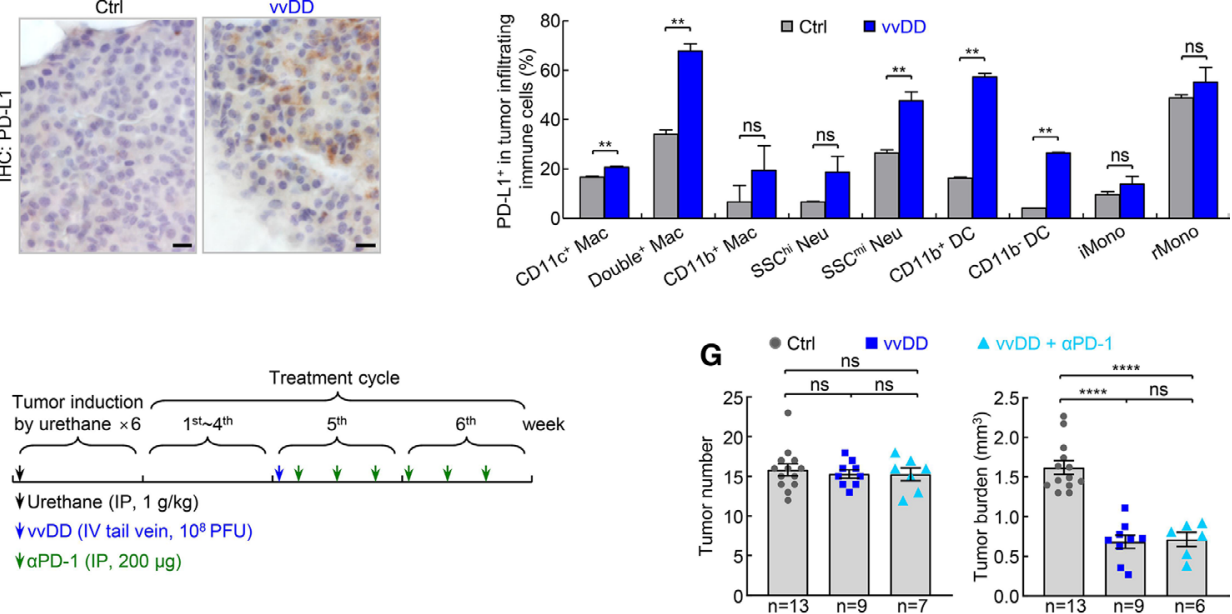

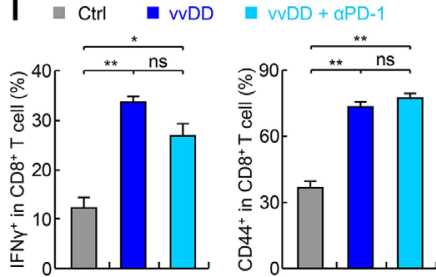

Figure 4 PD-1 blockade fails to enhance vvDD's efficacy in treating refractory lung cancer. (A) FACS assays showing increased pulmonary PD $-1^{+} \mathrm{CD} 4^{+}$and $\mathrm{CD} 8^{+} \mathrm{T}$ cells by vvDD treatment in urethane model $(n=3)$. (B) FACS assays showing increased tumor-infiltrating PD- $1^{+} C D 8^{+}$T cells by vvDD treatment in urethane model $(n=5)$. (C) FACS assays showing increased PD-L1 expression on tumor cells by vVDD treatment in urethane model $(n=3)$. (D) IHC assays showing increased PD-L1 expression on tumor cells by vvDD treatment in urethane model. scale bar, $10 \mu \mathrm{m}$. (E) FACS assays showing increased PD-L1 expression on tumor-associated immune cells by vvDD treatment in urethane model $(n=3)$. (F) Tumor treatment schedule. (G) Urethane model showing no enhancement of VVDD efficacy in refractory lung cancer by PD-1 antibody. (H) IHC showing no enhancement of vvDD-induced tumor cell apoptosis by PD-1 antibody treatment in urethane model $(n=9)$. Scale bar, $10 \mu \mathrm{m}$. (I) FACS assays of IFN $\gamma$ and CD44 showing no significant increase in vVDD-induced CD8 ${ }^{+}$T-cell activation by PD-1 antibody treatment in urethane model $(n=3)$. DC, dendritic cell; FACS, fluorescent activated cell sorting; IFN $\gamma$, interferon- $\gamma$; hi, high; iMono, inflammatory monocyte; IHC, immunohistochemistry; Mac, macrophage; mi, middle; Neu, neutrophil; ${ }^{*} p<0.05,{ }^{* \star} p<0.01,{ }^{* \star *} p<0.001$, ${ }^{* * * *} \mathrm{p}<0.0001$; ns, not significant; PD-1, programmed cell death 1; PD-L1, programmed death ligand 1; rMono, resident monocyte; SSC, side scatter; vvDD, vaccinia virus with double deletion.

in the presence or absence of the vvDD treatment, suggesting high exhaustion of TILs, since PD- $1^{+} \mathrm{CD} 8^{+}$ $\mathrm{T}$ cells show decreased tumor cell killing ability (online supplementary additional file 1: online supplementary figure S5a). vvDD treatment also induced PD-L1 and PD-L2 expression on tumor cells and tumor-associated immune cells and CD11 $\mathrm{c}^{+}$alveolar macrophages in particular (figure 4C-E; online supplementary additional file 1: online supplementary figure S5b). These data suggested that induction of PD-1 expression on T cells and PD-L1 and PD-L2 on tumor cells and tumor-associated immune cells may limit the antitumor activity of vvDD. More importantly, these data together with data shown in figure 3 also suggested that vvDD can convert cold lung cancers into hot ones that may be sensitive to PD-1 blockade. To test this, we treated mice harboring endogenous lung cancers induced by urethane with both vvDD and PD-1 antibody (figure 4F). The additional treatment with PD-1 antibody had no effect on either tumor number or tumor burden, in comparison to vvDD treatment alone (figure 4G). The tumor cell apoptosis rates were also comparable in vvDD monotherapy and combination therapy (figure $4 \mathrm{H}$ ).

Surprisingly, PD-1 antibody treatment did not further enhance $\mathrm{CD}^{+}$T-cell activation (figure 4I), despite increased percentages of $\mathrm{PD}-1^{+} \mathrm{T}$ cells and increased PD-L1 and PD-L2 expression on tumor cells and tumor associated immune cells after vvDD treatment. Nevertheless, these data suggested that vvDD alone and/or in 
A

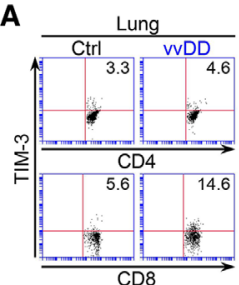

D

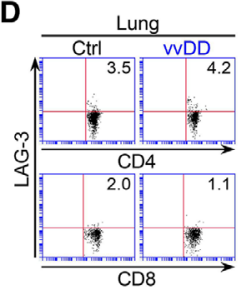

B
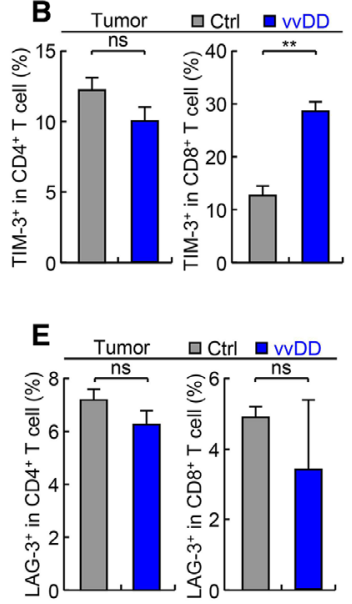
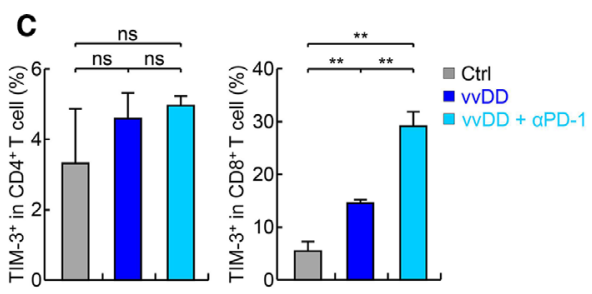

$\mathbf{F}$
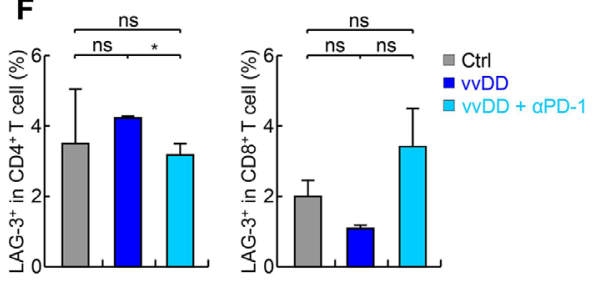

G
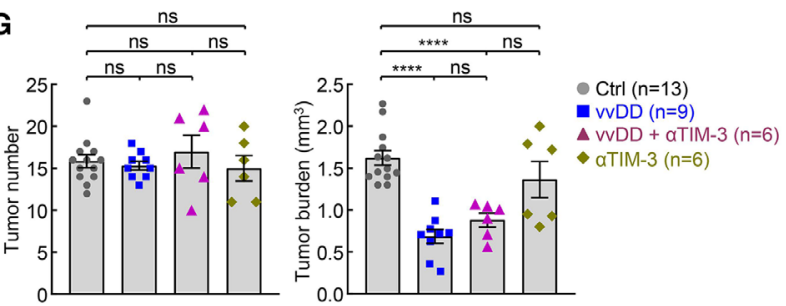

$\mathbf{H} \square \operatorname{Ctrl}(\mathrm{n}=7) \quad \square \mathrm{aTIM}-3(\mathrm{n}=3)$
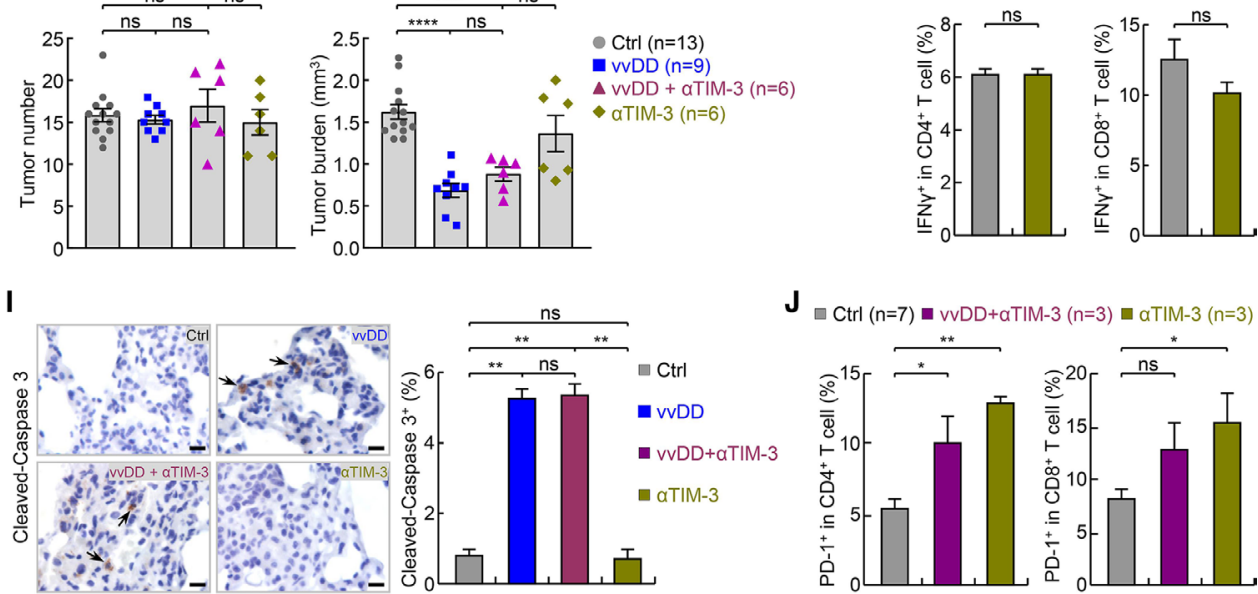

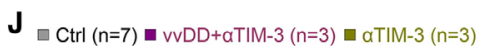

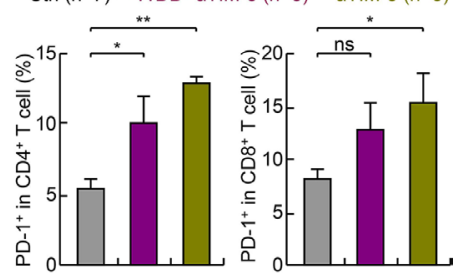

Figure 5 TIM-3 blockade shows no efficacy or synergy with vVDD in treating refractory lung cancer. (A) FACS assays showing increased pulmonary TIM- $3^{+} \mathrm{CD} 8^{+} \mathrm{T}$ cells by vvDD treatment in urethane model. (B) FACS assays showing increased tumorinfiltrating TIM $-3^{+} \mathrm{CD} 8^{+} \mathrm{T}$ cells but not TIM $-3^{+} \mathrm{CD} 4^{+} \mathrm{T}$ cells by vvDD treatment in urethane model $(\mathrm{n}=5)$. (C) FACS assays showing further increased pulmonary TIM $-3^{+} \mathrm{CD} 8^{+} \mathrm{T}$ cells but not TIM $-3^{+} \mathrm{CD} 4^{+} \mathrm{T}$ cells by VVDD/PD -1 antibody combination treatment in urethane model $(n=3)$. (D) FACS assays showing no changes in pulmonary LAG- $3^{+}$CD $4^{+}$and CD8 ${ }^{+}$T cells by vvDD treatment in urethane model. (E) FACS assays showing no significant changes in tumor-infiltrating LAG- $3^{+} \mathrm{CD} 4^{+}$and LAG- $3^{+} \mathrm{CD} 8^{+} \mathrm{T}$ cells by vvDD treatment in urethane model $(n=4)$. (F) FACS assays showing no changes in pulmonary LAG- $3^{+}$CD $4^{+}$and $C D 8^{+} \mathrm{T}$ cells by vvDD/PD-1 antibody combination treatment in urethane model $(n=3)$. (G) Urethane model showing no efficacy of TIM-3 blockade alone in treating refractory lung cancer or enhancing vvDD therapy. (H) FACS assays showing no significant changes in $\mathrm{CD}^{+}$and $\mathrm{CD}^{+}$T-cell activation by TIM-3 antibody treatment in urethane model. (I) IHC showing no enhanced tumor cell apoptosis by TIM-3 antibody treatment in urethane model $(n=9)$. Scale bar, $10 \mu \mathrm{m}$. (J) FACS assays showing increased PD-1 expression on $\mathrm{CD}^{+}$and $\mathrm{CD}^{+} \mathrm{T}$ cells by TIM-3 antibody treatment alone or together with vvDD in urethane model. FACS, fluorescent activated cell sorting; IHC, immunohistochemistry; ${ }^{*} p<0.05,{ }^{* *} p<0.01,{ }^{* * *} p<0.001,{ }^{* \star * *} p<0.0001$; ns, not significant; PD-1, programmed cell death 1; TIM-3, T-cell immunoglobulin and mucin-domain containing-3; vvDD, vaccinia virus with double deletion.

combination with PD-1 antibody also activates additional mechanism(s) other than PD-1/PD-L1/PD-L2 induction to restrict T-cell activation by vvDD. To this end, we examined whether wDD treatment induces expression of TIM-3 and lymphocyte-activation gene 3 (LAG-3, also known as CD223) on T cells. Like PD-1, TIM-3 and LAG-3 are well-known immune checkpoints that mediate T-cell exhaustion, particularly that of $\mathrm{CD}^{+} \mathrm{T}$ cells. ${ }^{232441-44} \mathrm{We}$ found that vvDD treatment significantly upregulated TIM-3 expression on $\mathrm{CD}^{+} \mathrm{T}$ cells but not $\mathrm{CD} 4^{+} \mathrm{T}$ cells in the lungs and in particular lung tumors (figure $5 \mathrm{~A}-\mathrm{C}$; online supplementary additional file 1: online supplementary figure S6a). Combination with PD-1 antibody further increased TIM-3 expression on $\mathrm{CD}^{+} \mathrm{T}$ cells (figure 5C). In addition, vvDD alone or together with PD-1 antibody significantly increased the expression of the TIM-3 ligands phosphatidylserine (PtdSer), carcinoembryonic antigen-related cell adhesion molecule 1 (CEACAM1, also known as CD66a) and high mobility group box 1 (HMGB1) on tumor cells and/or tumorassociated $\mathrm{CD} 11 \mathrm{c}^{+}$alveolar macrophages but had no effect on the expression of Galectin-9, another known ligand 
for TIM-3 (online supplementary additional file 1: online supplementary figures S4c, S6b-d). However, vvDD alone or together with PD-1 antibody had no effect on LAG-3 expression on either $\mathrm{CD} 8^{+}$or $\mathrm{CD} 4^{+} \mathrm{T}$ cells as well as the expression of the LAG-3 ligand liver and lymph node sinusoidal endothelial cell C-type lectin (LSECtin, also known as C-type lectin superfamily 4, member G, CLEC4G) on tumors (figure 5D-F; online supplementary additional file 1: online supplementary figure S7a), although those treatments induced the expression of fibrinogen-like protein 1 (FGL1) and MHC-II, two other known ligands of LAG-3, on tumor cells and tumor-infiltrating DCs and macrophages, respectively (online supplementary additional file 1: online supplementary figure $S 7 b, c)$.

Accordingly, we tested whether TIM-3 blockade enhances the therapeutic effect of vvDD. We also tested whether TIM-3 antibody alone has any therapeutic effect on refractory lung cancer, as a control. Of note, the role of TIM-3 blockade in lung cancer treatment has not been well defined yet. In line with the relatively low basal expression of TIM-3 on TILs (figure 5B), treatment with TIM-3 antibody had no significant therapeutic effect (figure 5G-I), suggesting that lung cancer is also largely intrinsically resistant to TIM-3 blockade. In this regard, our analysis of TCGA data indicated that TIM-3 expression was significantly downregulated in human lung tumor tissues compared with normal human lung tissues (online supplementary additional file 1: online supplementary figure S8). TIM-3 blockade also failed to enhance the therapeutic effect of voDD, although vwDD treatment could potently induce TIM-3 expression on $\mathrm{CD}^{+}$TILs. Mechanistically, we found that treatment with TIM-3 antibody alone or together with vvDD increased PD-1 expression on $\mathrm{CD}^{+}$and $\mathrm{CD} 8^{+} \mathrm{T}$ cells (figure $5 \mathrm{~J}$ ). These data together suggested that simultaneous induction/activation of PD-1 and TIM-3 by vDDD and mutual induction of PD-1 and TIM-3 by their blockade contribute to the restriction of vvDD's antitumor activity and the failure of synergy between wDD with single blockade of PD-1 or TIM-3 in lung cancer treatment.

\section{Improvement of vvDD viro-immunotherapy by dual blockade of PD-1 and TIM-3}

We next tested whether dual blockade of PD-1 and TIM-3 improves the efficacy of voD in refractory lung cancer in the endogenous lung cancer model, with a treatment schedule identical to figure $4 \mathrm{~F}$, with the addition of anti-TIM3 concurrent with anti-PD-1. Similar to single blockade, dual blockade of PD-1 and TIM-3 only showed minimal therapeutic effect (figure 6A). However, PD-1 and TIM-3 dual blockade combined with vvDD showed great synergy. In comparison to vvDD treatment alone, combination with both PD-1 and TIM-3 antibodies led to significantly reduced tumor burden, and importantly, significantly reduced tumor number, suggesting not only an overall tumor regression but also complete remission of some tumors. PD-1 and TIM-3 dual blockade also showed minimal therapeutic effect but great synergy when combined with vvDD in syngeneic lung cancer model, as evidenced by both tumor growth curve and animal survival rate (figure 6B,C). Dual blockade of PD-1 and TIM-3 also significantly increased activation of both $\mathrm{CD}^{+}$and $\mathrm{CD}^{+} \mathrm{T}$ cells and apoptosis of tumor cells by vvDD (figure 6D-F). These preclinical data suggest that combination of vvDD with dual blockade of PD-1 and TIM-3 may be effective for the treatment of human lung cancers, particularly those resistant to PD-1/PD-L1 or TIM-3 blockade.

\section{DISCUSSION}

Lung cancer is the no. 1 cancer killer, with about 234000 new cases and 154000 deaths yearly in the USA alone. ${ }^{45}$ Recently, PD-1/PD-L1 blockade immunotherapy has been approved for treatment of lung and several other cancers. ${ }^{1-10}$ While some patients have shown dramatic responses, the majority of patients do not benefit. Our human data studies indicate that PD-L1 is upregulated in only a minority of cancer cases, and downregulated in the majority of lung cancers. Moreover, T-cell activation within the TME is downregulated in most human lung cancers. These may account for the low response rate of PD-1 pathway blockade therapy in lung cancer patients. Paradoxically, PD-1 is upregulated in most human lung cancers (online supplementary additional file 1: online supplementary figure S9). Applying an endogenous mouse lung cancer model that closely resembles human lung cancer in genetics, molecular and immunological biology, as well as morphology and histology, ${ }^{27-29} 373940$ we observed PD-L1 downregulation and low TILs with increased PD-1 expression as well as overall resistance to PD-1 blockade therapy similar to humans. Using this preclinical model, we show that OVs can be used to treat refractory lung cancers as monotherapy, and more importantly, to turn cold lung tumors hot for better combination treatment.

In addition to inducing tumor cell lysis to trigger tumor-specific T-cell recruitment/infiltration and activation for further tumor cell killing, OVs stimulate PD-1 and TIM-3 expression on T cells as well as the expression of PD-1 and TIM-3 ligands on tumor cells and tumorassociated immune cells, inhibiting antitumor T-cell activation. Consistent with their redundant function in T-cell repression, blockade of either PD-1 or TIM-3 alone fails to enhance the therapeutic efficacy of OVs. PD-1 blockade further increases TIM-3 expression on T cells, and TIM-3 blockade alone or together with OVs induces PD-1 expression on T cells, providing rationale for the failure to combine OVs with either PD-1 or TIM-3 antibody individually for improved therapeutic efficacy. Simultaneous blockade of both PD-1 and TIM-3 significantly enhances T-cell activation and therapeutic efficacy of OVs, suggesting a new combination therapy for lung and other refractory cancers.

Given that upregulation of the PD-1 and TIM-3 pathways underlies viral immune escape, ${ }^{24}{ }^{41}$ although increasing 
A
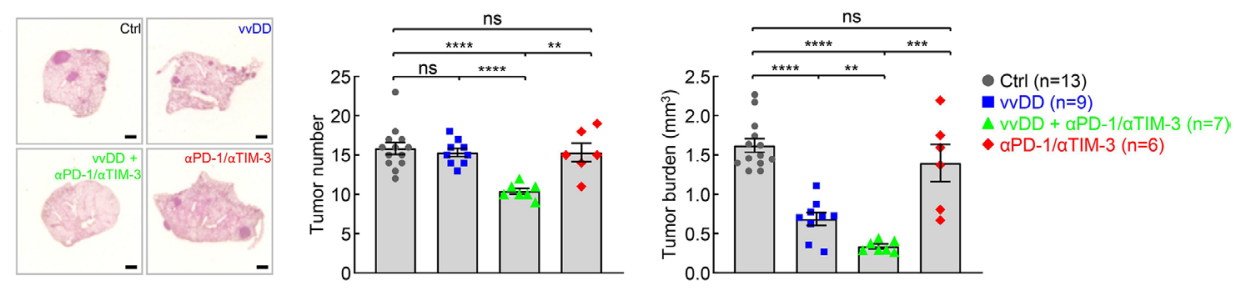

B
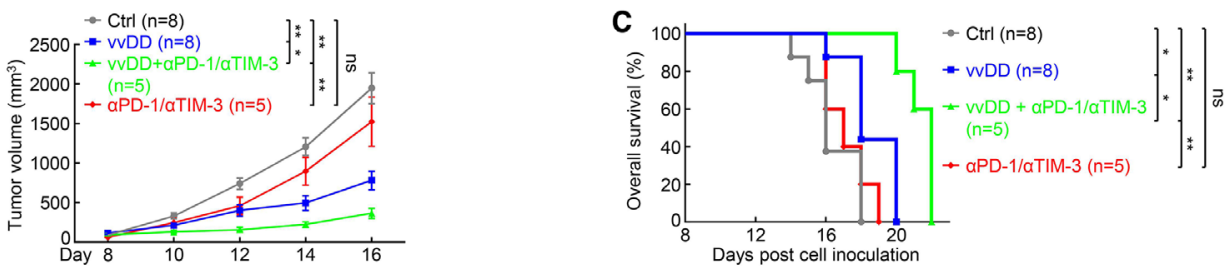

D
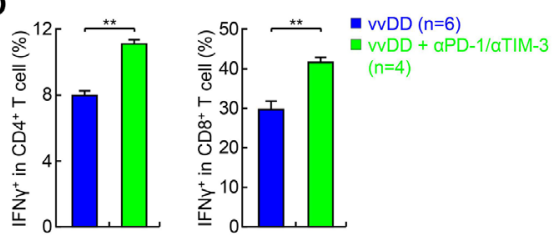

$E$
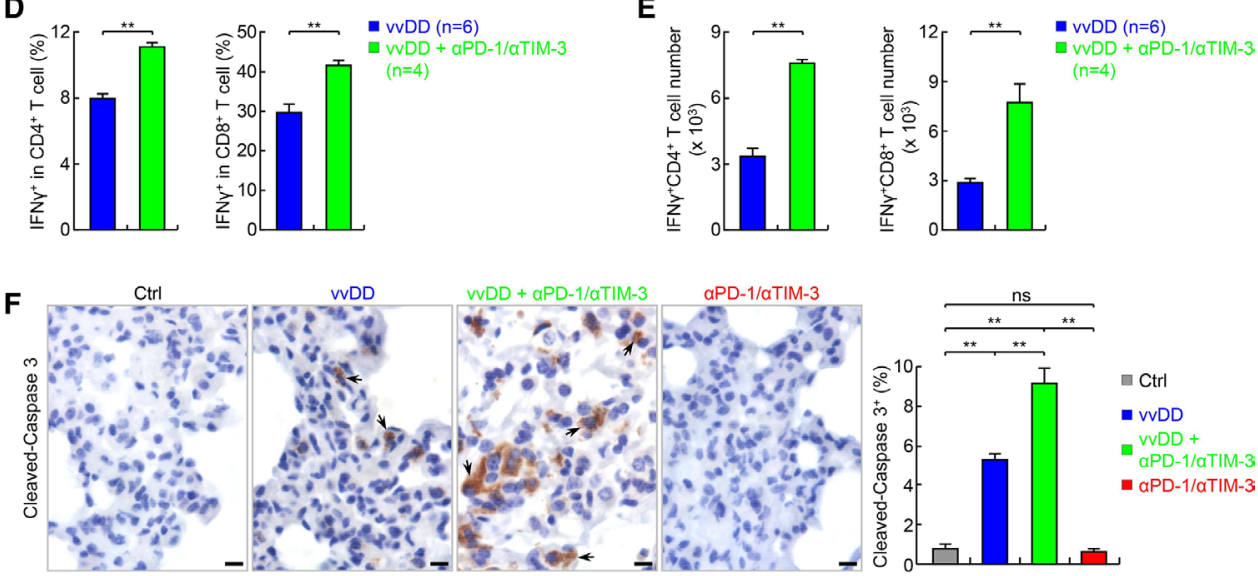

Figure 6 Dual blockade of PD-1 and TIM-3 synergizes with vVDD in treating refractory lung cancer. (A) Urethane model showing no significant efficacy of PD-1 and TIM-3 dual blockade alone but a synergy with vVDD in treating refractory lung cancer. scale bar, $1 \mathrm{~mm}$. (B, C) Tumor growth (B) and mouse survival analysis (C) showing no significant efficacy of PD-1 and TIM-3 dual blockade alone but a synergy with VvDD in syngeneic LAP0297 lung cancer model. Note, mice died before day 16 in the control (Ctrl) group were not included for the tumor volume calculation at the day 16 timepoint. (D) FACS assays of IFN $\gamma$ showing increase in the percentage of activated pulmonary $\mathrm{CD} 4^{+}$and $\mathrm{CD} 8^{+} \mathrm{T}$ cells by combination therapy in urethane model. (E) FACS assays showing increase in the number of activated pulmonary CD4 ${ }^{+}$and $\mathrm{CD}^{+} \mathrm{T}^{\mathrm{T}}$ cells by combination therapy in urethane model. (F) IHC showing increased tumor cell apoptosis by combination therapy in urethane model ( $n=9)$. Scale bar, $10 \mu \mathrm{m}$. FACS, fluorescent activated cell sorting; IFN $\gamma$, interferon $\gamma$; IHC, immunohistochemistry; ${ }^{*} p<0.05,{ }^{* *} p<0.01,{ }^{\star \star *} \mathrm{p}<0.001$, ${ }^{\star \star * \star} \mathrm{p}<0.0001$; ns, not significant; PD-1, programmed cell death 1; TIM-3, T-cell immunoglobulin and mucin-domain containing-3; vvDD, vaccinia virus with double deletion.

antitumor immunity, blockade of PD-1 and TIM-3 may also release the brake on the antiviral immune response, speeding up virus clearance. Thus, it is better not to start to administer PD-1 and TIM-3 antibodies simultaneously with or immediately after OV administration. This will allow OVs to replicate, spread, and enhance tumor killing prior to enhanced viral clearance from PD-1 and TIM-3 blockade. Our studies also indicate that PD-1 and TIM-3 antibodies should be used together and simultaneously, because they both are induced by OVs, and importantly, blockade of one of them is not only ineffective but also leads to upregulation of the other one on T cells, leading to T-cell suppression and diminution of antitumor activity.

Mouse studies on OVs mainly use syngeneic models. Although informative, syngeneic models are not ideal for studying cancer immunology and immunotherapy, because they do not resemble the natural co-evolution of tumor and immune cells. ${ }^{46}$ They can induce acute immune response with fundamental differences from chronic immune responses in endogenous tumors. For example, MAD109 lung cancer cells, which were originally obtained from a cold tumor with minimal PD-L1 expression, can form hot tumors with high PD-L1 expression and infiltrating $\mathrm{T}$ cells after being s.c. inoculated into syngeneic mice (online supplementary additional file 1: online supplementary figure S10). Syngeneic tumor models may also show some fundamental differences in response to OVs, although they, like the endogenous mouse lung tumor model, may increase PD-1 expression on T cells and PD-L1 on tumor cells and tumor-associated immune cells after OV intratumoral injection (online supplementary additional file 1: online supplementary figure S11a, b). Unlike in the endogenous lung tumor model, OV administration does not upregulate but instead decreases the percentages of TIM- $3^{+}$TILs in the MAD109 syngeneic model. Accordingly, in contrast to 
the endogenous urethane lung tumor model described here, PD-1 blockade and OVs show therapeutic synergy in this syngeneic lung tumor model as well as in syngeneic colon and ovarian tumors. ${ }^{47-50}$ The upregulation of TIM-3 in the presence of OVs as seen in the endogenous model may represent a more relevant physiologic scenario, as upregulation of both PD-1 and TIM-3 on T cells by several different viruses has been described under non-tumor conditions. ${ }^{23} 24$ 41-44 Our studies also suggest that the LAP0297 syngeneic lung tumor model may be a better alternative for studying refractory lung cancers compared with the MAD109 model, as it, like the endogenous lung cancer model, also shows TIM-3 induction on $\mathrm{CD}^{+}$TILs by OV administration (online supplementary additional file 1: online supplementary figure S11c, d).

\section{CONCLUSIONS}

In summary, we find that PD-L1 and T-cell activation are downregulated in a majority of human lung cancers, and that urethane-induced lung tumor in mice is a reliable endogenous model of human lung cancer with decreased PD-L1 expression and low TILs and innate resistance to PD-1/PD-L1 blockade. Using this preclinical model of lung cancer and various in vivo, ex vivo and in vitro assays, we find that intravenous administration of OVs promotes tumor cell death by the dual mechanisms of direct tumor cell killing and inducing T-cell tumor infiltration and activation, along with increasing expression of PD- 1 and TIM- 3 on T cells and PD- 1 and TIM-3 ligands on tumor cells and tumor-associated immune cells. We have described here that single blockade of either PD-1 or TIM-3, particularly when being combined with OV treatment, increases the expression of the other one on $\mathrm{T}$ cells. These findings help to characterize lung cancer resistance to oncolytic viro-immunotherapy combined with either PD-1 or TIM-3 blockade alone. However, OVs combined with both PD-1 and TIM-3 blockade were highly effective in our model, providing a strong rationale for the triple combination therapy for refractory lung cancer and possibly other cancer types, particularly those cold tumors otherwise resistant to treatment.

Acknowledgements The authors thank A.L. Epstein, P.H. Basse/S.H. Thorne, and D. Fukumura/P. Huang for providing MAD109, LLC cells and LAP0297, respectively. The authors also thank Y. Xiao and X. Chu for technical assistance.

Contributors $Z Q$ and $G X$ conceived and designed the study, led and contributed to all aspects of the analysis, and wrote the manuscript. FS performed the experiments. ZSG provided the oncolytic vaccinia virus and advice. ADG and SDS provided advice and constructive feedback.

Funding This study was supported in part by the National Institute of Health (NIH)/ National Cancer Institute (NCl) grant R21 CA189703, University of Pittsburgh Center for AIDS Research and University of Pittsburgh Schools of the Health Sciences Bridge funding (ZQ), NIH/NCl R01 CA172090, American Lung Association (ALA) Lung Cancer Discovery Award LCD 259111 and University of Pittsburgh Schools of the Health Sciences Bridge funding (GX) and Flight Attendant's Medical Research Institute YFAC142013 (ADG)

Competing interests No, there are no competing interests.

Patient consent for publication Not required.
Ethics approval All animal studies were approved by the Institutional Animal Care and Use Committee of the University of Pittsburgh and carried out in accordance with guidelines of the National Institutes of Health on animal care.

Provenance and peer review Not commissioned; externally peer reviewed.

Data availability statement All data relevant to the study are included in the article or uploaded as supplementary information. All data generated or analyzed during this study are included in this article and its additional files.

Open access This is an open access article distributed in accordance with the Creative Commons Attribution Non Commercial (CC BY-NC 4.0) license, which permits others to distribute, remix, adapt, build upon this work non-commercially, and license their derivative works on different terms, provided the original work is properly cited, appropriate credit is given, any changes made indicated, and the use is non-commercial. See http://creativecommons.org/licenses/by-nc/4.0/.

\section{ORCID iD}

Zhaoxia Qu http://orcid.org/0000-0002-2769-9814

\section{REFERENCES}

1 Bardhan K, Anagnostou T, Boussiotis VA. The PD1:PD-L1/2 pathway from discovery to clinical implementation. Front Immunol 2016;7:550.

2 Topalian SL, Drake CG, Pardoll DM. Immune checkpoint blockade: a common denominator approach to cancer therapy. Cancer Cell 2015;27:450-61.

3 Malhotra J, Jabbour SK, Aisner J. Current state of immunotherapy for non-small cell lung cancer. Trans/ Lung Cancer Res 2017;6:196-211.

4 Chen L, Han X. Anti-Pd-1/Pd-L1 therapy of human cancer: past, present, and future. J Clin Invest 2015;125:3384-91.

5 Nishino M, Ramaiya NH, Hatabu H, et al. Monitoring immunecheckpoint blockade: response evaluation and biomarker development. Nat Rev Clin Oncol 2017;14:655-68.

6 Vonderheide $\mathrm{RH}$. The immune revolution: a case for priming, not checkpoint. Cancer Cell 2018;33:563-9.

7 Zappasodi R, Merghoub T, Wolchok JD. Emerging concepts for immune checkpoint blockade-based combination therapies. Cancer Cell 2018;33:581-98.

8 Vokes EE, Ready N, Felip E, et al. Nivolumab versus docetaxel in previously treated advanced non-small-cell lung cancer (CheckMate 017 and CheckMate 057): 3-year update and outcomes in patients with liver metastases. Ann Oncol 2018;29:959-65.

9 Reck M, Rodríguez-Abreu D, Robinson AG, et al. Pembrolizumab versus chemotherapy for PD-L1-positive non-small-cell lung cancer. N Engl J Med 2016;375:1823-33.

10 Gettinger S, Horn L, Jackman D, et al. Five-Year follow-up of nivolumab in previously treated advanced non-small-cell lung cancer: results from the CA209-003 study. J Clin Oncol 2018;36:1675-84.

11 Kaufman HL, Kohlhapp FJ, Zloza A. Oncolytic viruses: a new class of immunotherapy drugs. Nat Rev Drug Discov 2015;14:642-62.

12 Martin NT, Bell JC. Oncolytic virus combination therapy: killing one bird with two stones. Mol Ther 2018;26:1414-22.

13 Guo ZS, Lu B, Guo Z, et al. Vaccinia virus-mediated cancer immunotherapy: cancer vaccines and oncolytics. J Immunother Cancer 2019;7:6.

14 Chaurasiya S, Chen NG, Fong Y. Oncolytic viruses and immunity. Curr Opin Immunol 2018;51:83-90.

15 Andtbacka RHI, Kaufman HL, Collichio F, et al. Talimogene laherparepvec improves durable response rate in patients with advanced melanoma. J Clin Oncol 2015;33:2780-8.

16 Zeh HJ, Downs-Canner S, McCart JA, et al. First-In-Man study of Western reserve strain oncolytic vaccinia virus: safety, systemic spread, and antitumor activity. Mol Ther 2015;23:202-14.

17 Downs-Canner S, Guo ZS, Ravindranathan R, et al. Phase 1 study of intravenous oncolytic poxvirus (VvDD) in patients with advanced solid cancers. Mol Ther 2016;24:1492-501.

18 Barber DL, Wherry EJ, Masopust D, et al. Restoring function in exhausted CD8 T cells during chronic viral infection. Nature 2006;439:682-7.

19 Mühlbauer M, Fleck M, Schütz C, et al. Pd-L1 is induced in hepatocytes by viral infection and by interferon-alpha and -gamma and mediates T cell apoptosis. J Hepatol 2006;45:520-8.

20 Green MR, Rodig S, Juszczynski P, et al. Constitutive AP-1 activity and EBV infection induce PD-L1 in Hodgkin lymphomas and posttransplant lymphoproliferative disorders: implications for targeted therapy. Clin Cancer Res 2012;18:1611-8. 
21 Topalian SL, Taube JM, Anders RA, et al. Mechanism-Driven biomarkers to guide immune checkpoint blockade in cancer therapy. Nat Rev Cancer 2016;16:275-87.

22 McCart JA, Ward JM, Lee J, et al. Systemic cancer therapy with a tumor-selective vaccinia virus mutant lacking thymidine kinase and vaccinia growth factor genes. Cancer Res 2001;61:8751-7.

23 Anderson AC. Tim-3: an emerging target in the cancer immunotherapy landscape. Cancer Immunol Res 2014;2:393-8.

24 Das M, Zhu C, Kuchroo VK. Tim-3 and its role in regulating antitumor immunity. Immunol Rev 2017;276:97-111.

25 Lechner MG, Karimi SS, Barry-Holson K, et al. Immunogenicity of murine solid tumor models as a defining feature of in vivo behavior and response to immunotherapy. J Immunother 2013;36:477-89.

26 Huang P, Duda DG, Jain RK, et al. Histopathologic findings and establishment of novel tumor lines from spontaneous tumors in FVB/N mice. Comp Med 2008;58:253-63.

27 Sun F, Qu Z, Xiao Y, et al. NF-kB1 p105 suppresses lung tumorigenesis through the Tpl2 kinase but independently of its NFкB function. Oncogene 2016;35:2299-310.

28 Li L, Han L, Sun F, et al. NF-kB RelA renders tumor-associated macrophages resistant to and capable of directly suppressing $C D 8^{+}$ T cells for tumor promotion. Oncoimmunology 2018;7:e1435250.

29 Zhou J, Qu Z, Yan S, et al. Differential roles of STAT3 in the initiation and growth of lung cancer. Oncogene 2015;34:3804-14.

30 Yan P, Fu J, Qu Z, et al. Pdlim2 suppresses human T-cell leukemia virus type I Tax-mediated tumorigenesis by targeting Tax into the nuclear matrix for proteasomal degradation. Blood 2009;113:4370-80

$31 \mathrm{Qu}$ Z, Fu J, Ma H, et al. Pdlim2 restricts Th1 and Th17 differentiation and prevents autoimmune disease. Cell Biosci 2012;2:23.

32 Sun F, Xiao Y, Qu Z. Oncovirus Kaposi sarcoma herpesvirus (KSHV) represses tumor suppressor PDLIM2 to persistently activate nuclear factor $\kappa \mathrm{B}(\mathrm{NF}-\mathrm{\kappa B})$ and STAT3 transcription factors for tumorigenesis and tumor maintenance. J Biol Chem 2015;290:7362-8.

33 Qu Z, Sun F, Zhou J, et al. Interleukin-6 prevents the initiation but enhances the progression of lung cancer. Cancer Res 2015;75:3209-15.

$34 \mathrm{Fu}$ J, Qu Z, Yan P, et al. The tumor suppressor gene WWOX links the canonical and noncanonical NF-kB pathways in HTLV-I Tax-mediated tumorigenesis. Blood 2011;117:1652-61.

$35 \mathrm{Qu} \mathrm{Z}$, Fu J, Yan P, et al. Epigenetic repression of PDZ-LIM domaincontaining protein 2: implications for the biology and treatment of breast cancer. J Biol Chem 2010;285:11786-92.

36 Cheng $\mathrm{H}$, Borczuk $\mathrm{A}$, Janakiram $\mathrm{M}$, et al. Wide expression and significance of alternative immune checkpoint molecules, B7x and
HHLA2, in PD-L1-negative human lung cancers. Clin Cancer Res 2018;24:1954-64.

37 Sun F, Li L, Yan P, et al. Causative role of PDLIM2 epigenetic repression in lung cancer and therapeutic resistance. Nat Commun 2019;10:5324.

38 Pin A, Monasta L, Taddio A, et al. An easy and reliable strategy for making type I interferon signature analysis comparable among research centers. Diagnostics 2019;9:113.

39 Tuveson DA, Jacks T. Modeling human lung cancer in mice: similarities and shortcomings. Oncogene 1999;18:5318-24.

40 Zhou J, Qu Z, Sun F, et al. Myeloid STAT3 promotes lung tumorigenesis by transforming tumor immunosurveillance into tumorpromoting inflammation. Cancer Immunol Res 2017;5:257-68.

41 Anderson AC, Joller N, Kuchroo VK. LAG-3, Tim-3, and TIGIT: coinhibitory receptors with specialized functions in immune regulation. Immunity 2016;44:989-1004.

42 Jones RB, Ndhlovu LC, Barbour JD, et al. Tim-3 expression defines a novel population of dysfunctional T cells with highly elevated frequencies in progressive HIV-1 infection. $J$ Exp Med 2008;205:2763-79.

43 Takamura S, Tsuji-Kawahara S, Yagita $\mathrm{H}$, et al. Premature terminal exhaustion of Friend virus-specific effector CD8+ T cells by rapid induction of multiple inhibitory receptors. $\mathrm{J}$ Immunol 2010;184:4696-707.

44 Golden-Mason L, Palmer BE, Kassam N, et al. Negative immune regulator Tim-3 is overexpressed on T cells in hepatitis $\mathrm{C}$ virus infection and its blockade rescues dysfunctional $\mathrm{CD}^{+}$and $\mathrm{CD}^{+} \mathrm{T}$ cells. J Virol 2009;83:9122-30.

45 Siegel RL, Miller KD, Jemal A. Cancer statistics, 2019. CA Cancer J Clin 2019;69:7-34.

46 Ostrand-Rosenberg S. Animal models of tumor immunity, immunotherapy and cancer vaccines. Curr Opin Immunol 2004;16:143-50.

47 Yan $\mathrm{X}$, Wang $\mathrm{L}$, Zhang $\mathrm{R}$, et al. Overcoming resistance to anti-PD immunotherapy in a syngeneic mouse lung cancer model using locoregional virotherapy. Oncoimmunology 2017;7:e1376156.

48 Liu Z, Ravindranathan R, Kalinski P, et al. Rational combination of oncolytic vaccinia virus and PD-L1 blockade works synergistically to enhance therapeutic efficacy. Nat Commun 2017;8:14754.

49 Kowalsky SJ, Liu Z, Feist M, et al. Superagonist IL-15-armed oncolytic virus elicits potent antitumor immunity and therapy that are enhanced with PD-1 blockade. Mol Ther 2018;26:2476-86.

50 Liu Z, Ge Y, Wang H, et al. Modifying the cancer-immune set point using vaccinia virus expressing re-designed interleukin-2. Nat Commun 2018:9:4682. 